\title{
Development of Data Processing Skills of Physics Students in Intermediate Laboratory Course
}

Kontro, Inkeri

Springer

2019

Kontro , I 2019 , Development of Data Processing Skills of Physics Students in Intermediate Laboratory Course . in E McLoughlin \& P van Kampen (eds), Concepts, Strategies and Models to Enhance Physics Teaching and Learning . Springer , Cham , pp. 101-108 . https://doi.org/10.1007/978-3-C

http://hdl.handle.net/10138/332575

https://doi.org/10.1007/978-3-030-18137-6_9

unspecified

acceptedVersion

Downloaded from Helda, University of Helsinki institutional repository.

This is an electronic reprint of the original article.

This reprint may differ from the original in pagination and typographic detail.

Please cite the original version. 


\title{
Development of Data Processing Skills of Physics Students on Intermediate Laboratory Courses
}

\author{
Inkeri Kontro $^{1[0000-0002-1874-3756]}$ \\ ${ }^{1}$ Department of Physics, University of Helsinki, POB 64, FI-00014 Helsinki \\ inkeri.kontro@helsinki.fi
}

\begin{abstract}
Laboratory courses are an essential part of physics education, and the focus on laboratory skills has recently been emphasized. We evaluated the effect of skills-oriented laboratory courses on data processing skills with the Concise Data Processing Assessment (CDPA). Though no overall improvement occurred, changes in answering patterns in CDPA occurred. The students showed favourable shifts in their answer patterns for items dealing with fit-ting error but small declines in identifying power laws in data. This is likely due to course curriculum, which emphasized fitting. Although the sample size is small, the results indicate that CDPA answers can provide information on learning of specific topics even if changes in the overall score are mini-mal. The measured data processing skills did not correlate with expert-like attitudes (as measured by the Colorado Learning Attitudes about Science Sur-vey for Experimental Physics) or course grades.
\end{abstract}

Keywords: Undergraduate, laboratory, student attitudes, data processing.

\section{Introduction}

Laboratory work is an essential part of most university physics education. However, traditional laboratory work does not foster conceptual understanding [1] and laboratory works are most useful in teaching students skills rather than physical concepts, which is why the American Association of Physics Teachers recommends assigning skillsbased learning goals to laboratory courses [2]. One important skill to learn is data processing and error estimation. The ability to understand the reliability of data is a fundamental skill for any scientist, and for physicists, a natural surrounding for learning this skill is the laboratory where the data is produced. However, there are few standardized tests for measuring data processing skills. One such test is the Concise Data Processing Assessment (CDPA) [3], which is a multiple-choice test that consists of ten questions. In CDPA, first-year students typically score points corresponding to pure guess $(23.5 \%)$ and faculty score on average $80 \%$ [3].

In addition to skills, interest in the PER community has recently focused on the development of expert-like attitudes during laboratory courses. Use of the Colorado Learning Attitudes about Science Survey for Experimental Physics (E-CLASS) [4] has 
shown that generally, traditional laboratory courses have a detrimental effect on the expert-like attitudes, whereas courses that focus on skills see small improvements [5].

The intermediate (2nd to 3rd year) laboratory courses at the University of Helsinki are a pair of half-semester long courses, which span the spring term. The courses have been reformed with a focus on learning goals [6]. The reform led to a high attainment of learning goals. While the students attained the learning goals of the courses, expertlike attitudes (as measured by E-CLASS) remained unchanged [6]. The teachers and students were satisfied with the course, but to study the relationship between learning, student attitudes and student experience further, we administered the CDPA survey and the E-CLASS survey, and compared the results to course grades. The goal was to see whether favourable attitudes towards experimental physics correlate with data processing skills before or after instruction. Their correlation with the grades were also measured. The grade summarizes the learning goals of the course, which includes but is not limited to data analysis skills and expert-like practices in the laboratory. The research questions are thus whether the standardized instruments measure learning in this course, and whether the course learning goals reflect all of the aspects to which we aspire.

\section{Data collection and analysis}

The responses to the surveys were collected as part of the course calculation exercises, and credit corresponding to a single problem was awarded for participation. Only the responses of the participants who gave consent for use of their responses in research are included in the analysis. The response rate for each questionnaire was high, 70$90 \%$. The amount of students who answered both CDPA questionnaires was 26, and students who answered both CDPA questionnaires and the E-CLASS pre-test was 22.

The CDPA survey was administered during the first week of teaching and after the mid-term break, at the beginning of the second course, when the relevant material had been covered. The E-CLASS surveys were administered during the second week of the first course and at the end of the second course. The surveys were spaced out to reduce the burden on a single week.

The CDPA was scored simple awarding 1 point for each correct answer. The normalized gain $g$ of CDPA was calculated from

$$
g=\frac{\text { post-pre }}{100-\text { pre }}
$$

where post and pre are the scores of the post- and pre-test, respectively [7].

The E-CLASS survey was asked both with the formulation "What do you do when performing an experiment" and "What would an experimental physicist say". The ECLASS survey was marked by collapsing the 5-point Likert scale (strongly agree strongly disagree) into categories of agree, disagree and neutral with respect to the expert answer, and assigning 1, -1 and 0 points for these response types, respectively [4].

In addition to the survey results, the data set consists of students' grades from the first course. These grades are overall grades of the course; grades of individual learning 
goals were not available. Grades were administered on a scale of $0-5$, where 0 is fail, 1 the lowest passing grade and 5 the highest grade. No students achieved grades of $0-1$ on this course.

\section{RESULTS AND DISCUSSION}

\subsection{1 Student understanding of error}

The average CDPA students on the intermediate laboratory course was $(4.04 \pm 0.41)$ in the post-test. Day and Bonn reported values of 3.03 and 4.73 for second and fourth year students, respectively [3]. The students on the intermediate laboratory course are generally second- to third year students, but some are older.

The CDPA score showed no change with instruction. The results from the pre- and post-tests are shown in Table 1 . The item difficulty varied significantly, and largely followed that presented in [3]: items 1, 5 and 6 were the most difficult. The only major deviance from this was the difficulty of item 4, in which the University of Helsinki students did more poorly than could be expected. This item concerns a log-log plot with a power law.

Table 1. CDPA scores $(\mathrm{N}=26)$, average score. Overall score with standard error of the mean and scores for individual questions pre- and post instruction, gain and normalized gain $g$.

\begin{tabular}{lcccc}
\hline Item & Pre & \multicolumn{2}{c}{ Post } & Gain \\
\hline Overall & $3.92 \pm 0.35$ & $4.04 \pm 0.41$ & $0.12 \pm 0.35$ & $-0.01 \pm 0.06$ \\
Question 1 & 0.23 & 0.31 & 0.08 & \\
Question 2 & 0.77 & 0.77 & 0.00 & \\
Question 3 & 0.31 & 0.35 & 0.04 & \\
Question 4 & 0.27 & 0.31 & 0.04 & \\
Question 5 & 0.08 & 0.19 & 0.11 & \\
Question 6 & 0.12 & 0.12 & 0.00 & \\
Question 7 & 0.62 & 0.46 & -0.16 & \\
Question 8 & 0.58 & 0.46 & -0.12 & \\
Question 9 & 0.77 & 0.69 & -0.08 & \\
Question 10 & 0.35 & 0.35 & 0.00 & \\
\hline
\end{tabular}

While no change in the overall score emerged, some answering patterns warrant a closer look.

The two most difficult questions, items 5 and 6, concern linear fits of data with error bars of varying size. The improvement for the correct choice was modest for question 5 and zero for question 6 , but there was a marked shift in the answers (Table 2). The right answer, naturally, was to fit the line to best match the data points with the smallest 
error bars (choices $5 \mathrm{~b}$ and $6 \mathrm{a}$, respectively). The answer choices were as follows (for question 5)

- a) weighing of more uncertain data points

- b) (correct) weighing of more precise data points

- c) unweighted fit

- d) intermediate fit weighing more precise data points somewhat more than the uncertain ones

For question 6, the answer choices were

- a) (correct) weighing the most precise data points

- b) unweighted fit

- c) touching the most error bars

- d) fitting an equal amount of data points below and above the line

Prior to instruction the most popular choice for question 5 was the unweighted fit $(5 \mathrm{c}$, 17 answers), with the moderately weighted choice (5d, 7) being second. Post instruction, the percentages were almost reversed (6 and 14 answers for $5 \mathrm{c}$ and $5 \mathrm{~d}$, respectively). In addition, the amount of correct answers had increased slightly (Table 2).

For question 6, the pre-instruction favourite was equal amount of points over and under the fit $(6 \mathrm{~d}, \mathrm{~N}=16)$ with the fit touching most error bars being second $(6 \mathrm{c}, \mathrm{N}=$ $6)$, and the correct option third $(6 \mathrm{a}, \mathrm{N}=3)$. The prevalence of $6 \mathrm{c}$ increased in the posttest, though the changes in the answer distribution are less marked than for item 5.

The changes in the answer patterns in particular to question 5 suggest that while the students have not fully grasped the extent to which the differences in error of data points affect fitting, they are aware that the error bars play a role. Answers that consider error bars were more common after instruction.

Table 2. CDPA answers for items 5 and $6(N=26)$. The right answer is underlined and the wrong answer, which still considers error bars (for questions 5 and 6 ), is in italics.

\begin{tabular}{lllll}
\hline Question 5 & $\mathrm{a}$ & $\underline{\mathrm{b}}$ & $\mathrm{c}$ & $d$ \\
\hline pre-test & 0 & 2 & 17 & 7 \\
post-test & 1 & 5 & 6 & 14 \\
\hline Question 6 & $\underline{\mathrm{a}}$ & $\mathrm{b}$ & $c$ & $\mathrm{~d}$ \\
\hline pre-test & 3 & 1 & 6 & 16 \\
post-test & 3 & 3 & 11 & 9 \\
\hline
\end{tabular}

However, is it fair to interpret these shifts favourably, when there are questions with non-favourable changes? The questions with the largest losses in right answers concerns identifying a power law graph (question 7) and identifying a power law in a table (question 8). In both questions, the incorrect assumption of exponential relationship is somewhat more common in the post-test. The other wrong choices have the correct 
power law with incorrect units $(7 \mathrm{~d}, 8 \mathrm{~b}$ and $8 \mathrm{c})$. The answer distributions to these questions are shown in Table 3.

Table 3. CDPA answers for items 7 and $8(N=26)$. The right answer is underlined and the answers containing exponentials are in italics. Answers with incorrect units are marked with asterisks.

\begin{tabular}{lllll}
\hline Question 7 & $a^{*}$ & $b$ & $\underline{\mathrm{c}}$ & $\mathrm{d}^{*}$ \\
\hline pre-test & 4 & 5 & 16 & 1 \\
post-test & 3 & 9 & 12 & 2 \\
\hline Question 8 & $\underline{\mathrm{a}}$ & $\mathrm{b}^{*}$ & $\mathrm{c}^{*}$ & $d$ \\
\hline pre-test & 15 & 3 & 4 & 4 \\
post-test & 12 & 6 & 3 & 5 \\
\hline
\end{tabular}

Power laws do not feature in the intermediate laboratory courses, however, exponentials and (semi)logarithmic plots do. Post-instruction, students opted slightly more for exponentials, which shows they may have opted for familiarity. In addition, the skill to recognise power laws are not explicitly taught on the laboratory courses or any previous course (Szabolcs Galambosi, private communication) which may explain both the declining results of items 7 and 8 and the surprising difficulty of item 4 , which also concerns a power law.

The third question with a negative gain, item 9, concerns rounding and measurement error of a scale measuring the mass of several objects, when the mass of one was known. Here, students were more prone to overestimating the error in the post-test by conflating the rounding error and the resolution of a scale (Table 4). The answer choices to question 9 were, in order:

- a) (correct) propagation of rounding error

- b) conflating the uncertainty of the mass of one object to that of the resolution of the scale

- c) conflating the rounding error of a single measurement to the resolution of the instrument (considered by some experts to be correct)

- d) ignoring uncertainty entirely

- e) not treating the rounding uncertainty symmetrically.

Hence, some students changed their answer from correct to overestimating the error, according to the CDPA scoring. However, some experts also consider this overestimation $(9 \mathrm{c})$ the correct choice [3], as the error of a measurement device is usually larger than just the rounding error. Indeed, during the laboratory course, the students familiarized themselves with instrument manuals that consistently state that the instrumental error is a function of, and larger than the resolution. Scales did generally not feature the 
students' design of lab experiments, but the uncertainty of e.g. multimeters had to be considered in error calculations.

Table 4. CDPA answers for item $9(\mathrm{~N}=26)$. The right answer is underlined and the answer that considers measurement error larger than rounding error is in italics.

\begin{tabular}{lllll}
\hline Question 9 & $\underline{\mathbf{a}}$ & b & $\boldsymbol{c}$ & d \\
\hline pre-test & 20 & 3 & 3 & 0 \\
post-test & 18 & 1 & 7 & 0 \\
\hline
\end{tabular}

As such, the conclusion is that there are small signals that the instruction affected the students' answer both for better and for worse. In linear fits, the students were more likely to choose answers that considered error bars, whether or not to the necessary degree. However, non-exponential relationships were slightly increasingly identified as exponential. Of course, with such a small sample, in particular the smaller changes in answers are likely to be the product of chance. The large change in the answering pattern to item 5 likely indicates that students learned to consider the error bars during the course, even if they were not able to correctly interpret the necessary weighing.

\section{Correlation of expert-like attitude, experience and skill}

The correlations between E-CLASS (pre-test) and CDPA scores is presented in Table 5. The number of complete datasets dropped to 22, and dropped further, when the E-CLASS post-test was added. For this reason, the E-CLASS post-test is not considered here, as the reliability of any results would decline further.

Table 5. The correlation coefficient $r$ between CDPA pre-instruction and post-instruction scores, CDPA normalized gain $g$, E-CLASS scores for questions phrased "What do you think when doing labs" (E-CLASS own) and "What would an experimental physicist say" (E-CLASS phys) and grade. $N=22$.

\begin{tabular}{lrrrrrr}
\hline & \multicolumn{1}{l}{$\begin{array}{l}\text { CDPA } \\
\text { pre }\end{array}$} & $\begin{array}{l}\text { CPDA } \\
\text { post }\end{array}$ & $\begin{array}{c}\text { CDPA } \\
\boldsymbol{g}\end{array}$ & $\begin{array}{c}\text { E-CLASS } \\
\text { own }\end{array}$ & $\begin{array}{c}\text { E-CLASS } \\
\text { phys }\end{array}$ & Grade \\
\hline CDPA pre & 1 & & & & & \\
CPDA post & 0.50 & 1 & & & & \\
CDPA $g$ & -0.37 & 0.55 & 1 & & & \\
ECLASS own & 0.04 & 0.10 & 0.10 & & & \\
ECLASS phys & -0.10 & 0.24 & 0.27 & 0.45 & 1 & 1 \\
Grade & -0.03 & 0.04 & 0.09 & 0.27 & 0.21 & \\
\hline
\end{tabular}

Curiously, there seems to be no correlation between CDPA scores and neither the student's expert-like attitudes nor their perception of expert attitudes, as measured by the E-CLASS questionnaire. CDPA and E-CLASS results did also not correlate with 
the students' grades from the laboratory course (Table 5). This is surprising, as we expected students who understand error analysis better to achieve higher grades. On the other hand, no very low-achieving students attended the course, which may skew the results.

The grading rubrics on the course were based on learning goals and explicitly covered data processing. The absence of correlation between CDPA scores and grades may be due to obfuscation by the other learning goals, such as experiment design, report writing and programming skills. As previously discussed, the CDPA covers a variety of topics that did not feature on the course. The students on these courses generally reached the established learning goals and the students considered the course useful and challenging [6].

The low correlation between CDPA normalized gain and post-test scores $(r=0.55$, adjusted $\left.r^{2}=0.27\right)$ is statistically significant $(p<0.01)$ but simply explained by that the students, who improved their score during instruction, were more likely to do better on the post-test. The very modest negative correlation between CDPA pre-test score and gain, which could indicate that students were more likely to learn error analysis if their prior knowledge was higher, was not significant $(p>0.05)$.

\section{CONCLUSIONS}

Classifying and identifying the CDPA answers indicates that students on the intermediate laboratory courses paid more attention to the effect of measurement error in linear fits, a topic that featured heavily in the exercises on the courses. The students found questions involving power laws more difficult than expected. An examination of the curriculum shows that they are rarely exposed to data for which they need to find a suitable model without constraints, and power laws do not feature on courses except in specific contexts. This warrants a critical look on the curriculum.

Expert-like attitudes did not correlate with data processing skills or achieving the general learning goals of the course, as measured by student grades.

Overall, CDPA seems to provide useful information on specific improvements and problems in the students' knowledge even when changes in overall score are not present. As such, it is a useful tool for probing not only the level of student knowledge but common misconceptions.

\section{Acknowledgements}

The author thanks Dr. Szabolcs Galambosi and Olga Heino for discussion of the results, and O.H. and Dr. Tommi Kokkonen for the discussion of the translation of the CDPA. The financial support of the Waldemar von Frenckell foundation is gratefully acknowledged. 


\section{References}

1. Royuk, B. and Brooks, D.W.: Cookbook Procedures in MBL Physics Exercises. J Sci Educ Technol 12(3), 317-324 (2003). DOI: 10.1023/A:1025041208915

2. MacIsaac, D.: Report: AAPT Recommendations for the Undergraduate Physics Laboratory Curriculum, Phys. Teach. 53(4), 253-253 (2015). DOI: 10.1119/1.4914580.

3. Day, J. and Bonn, D.: Development of the Concise Data Processing Assessment. Phys Rev Spec Top-Phys Edu Res 7(1), 010114, (2011). DOI: 10.1103/PhysRevSTPER.7.010114

4. Zwickl, B.M., Finkelstein, N. and Lewandowski, H.J.: Development and Validation of the Colorado Learning Attitudes about Science Survey for Experimental Physics. In: Engelhardt, P.V., Churukian, A.D. and Rebello, N.S. (eds.) 2012 PHYSICS EDUCATION RESEARCH CONFERENCE, vol 1513, pp. 442-445. AIP, Philadelphia, PA, USA (2013) DOI: $10.1063 / 1.4789747$

5. Wilcox, B.R. and Lewandowski, H.J.: Open-ended versus guided laboratory activities: Impact on students' beliefs about experimental physics. Phys Rev Phys Edu Res 12(2) 020132. (2016) DOI: 10.1103/PhysRevPhysEducRes.12.020132

6. Kontro, I., Heino, O., Hendolin, I. and Galambosi, S. Modernisation of the intermediate physics laboratory. Eur. J. Phys. 39(2), 025702 (2018). DOI: 10.1088/1361-6404/aa9364

7. Hake, R.R.: Interactive-engagement versus traditional methods: A six-thousand-student survey of mechanics test data for introductory physics courses. Am J Phys 66(1), 64-74 (1998). DOI: $10.1119 / 1.18809$ 